\title{
Orthosis with a controllable plantar height for fall prevention using a compact magnetorheological fluid brake
}

\author{
Kohei MORIMURA ${ }^{1}$, Rieko YAMAMOTO ${ }^{1.2}$, and Toshihiko SHIRAISHI ${ }^{1 *}$ \\ 1) Graduate School of Environment and Information Sciences, Yokohama National University \\ 79-7, Tokiwadai, Hodogaya-ku, Yokohama, 240-8501, Japan
}

2) Department of Rehabilitation Medicine, Keio University School of Medicine, 35 Shinanomachi, Shinjuku-ku, Tokyo, 160-8582, Japan
E-mail: kmori554b.htoy@gmail.com (Kohei MORIMURA)
yamamoto-rieko-tz@ynu.jp (Rieko YAMAMOTO)
shira@ynu.ac.jp (Toshihiko SHIRAISHI)

${ }^{*}$ Corresponding author

\begin{abstract}
In this study, a compact magnetorheological (MR) fluid brake embedded in an orthosis was designed and fabricated to maintain plantar height for use in lateral fall prevention, and its performance and the fall prevention effect of the orthosis were evaluated. The results of the brake design and fabrication indicated that the MR fluid brake has the advantage of being compact, lightweight, and power-saving at $88.4 \mathrm{~mm}$ long, $128 \mathrm{~g}$, using two AA batteries. Performance tests of the brake indicated that it was able to generate sufficient force $(125 \mathrm{~N})$ to maintain plantar height. Furthermore, through the assessments of lateral fall, the center of gravity and center of pressure inclination angle of a subject wearing the orthosis without the MR brake was determined to be $4.0^{\circ}$, as opposed to $4.4^{\circ}$ with the MR fluid brake in the ON state. Therefore, the moment of force acting on the subject to prevent fall was larger, making them more stable when the MR fluid brake was in the ON state.
\end{abstract}

\section{Keywords}

Magnetorheological fluid, brake, controllable device, wearable device, fall prevention, plantar

\section{Introduction}

In recent years, hospital stays have shortened, and the number of people with disabilities living in the community has been increasing. Living in a community requires necessary activities such as shopping, hospital visits, etc., that require to walk on uneven surfaces, such as inclines and uneven surfaces outdoors, and on flat surfaces 
indoors. The elderly, hemiplegic patients, and cerebellar ataxia, who often show a decreased lateral stability, are particularly prone to losing their balance in the lateral direction during the stance phase on the affected side, leading to lateral falls. According to a study that examined fall direction, $34 \%$ of falls in elderly were lateral (Cummings and Nevitt, 1989).The higher risk of falling when walking on a lateral incline was found to be due to the increased activity of the peroneal muscles to maintain balance compared to walking on flat surface (Bavdek, Zdolsek, et al., 2018). The other factor is that the distance of the outward center of gravity (COG) movement is significantly smaller in the group that has experienced falling than in the group without experience (Barak, Wagennar, et al., 2006). Pelvis acceleration is significantly smaller in the elderly than in the young when walking on level and uneven grounds (Menz, Lord, et al., 2003); thus, the COG movement range is smaller in the elderly, leading to increased risk of lateral fall (Takai, Miyano, et al., 2001). A lateral fall would likely cause femoral neck fractures, which are severe and would likely require hospitalization. The risk of complications and mortality from immobility is high (WHO scientific group, 2003). Lateral fall prevention is an essential factor for maintaining safe mobility in their community and associated daily activity level that requires a specific solution. To this end, we consider herein a wearable device that can be used unobtrusively in the user's living environment and daily life, preventing falls only when a risk occurs.

The COG-center of pressure (COP) inclination angle is used as an index to evaluate the effect of fall prevention. Many studies have used the COG movement and the relative position of the COG to the COP to investigate the walking stability (MacKinnon and Winter, 1993; Kaya, Kreb, et al., 1998). Examples include the COG momentum (Kaya, Kreb, et al., 1998) and the maximum horizontal distance between COG and COP (Krebs, Gillbody, et al., 1993; Krebs, McGibbon, et al., 2001). However, these values may be affected by the subject's height (Berger, Trippel, et al., 1992); thus, it is necessary to have a gait stability index that can eliminate the variation among subjects. The angle between the line connecting COG and the COP and the vertical line passing through the COP (COG-COP inclination angle) has been proposed as an index. This angle may be used to evaluate the risk of falling (Lee and Chou, 2006). We focus on insole therapy to investigate methods of fall prevention. The plantar orthosis used in insole therapy is an orthotic device used to treat pain in osteoarthritis of the knee and big toe and prevent foot injuries in athletes. The main effects of foot orthosis have been reported from various biomechanical perspectives, such as correcting the alignment of the lower limb and the whole body, eliminating pain by load distribution, and improving the shock mitigation function during ground contact during walking (Kobayashi and Shimizu, 2014). When a plantar orthosis provides plantar height to the lateral side of the foot, the COP moves laterally, and the COG-COP inclination angle increases (Figure 1). In the case that a subject rotates with an angular velocity and the point where the combined force of gravity and inertia acting on the COG is projected onto the floor is inside the base of support (BOS) (Figure 1 left), the moment of force to prevent fall does not act on the subject leading to instability and a fall . On the other hand, in the case that a subject rotates with an angular velocity and the projected point described above exceeds the BOS limit and is in the opposite side to the rotation by a planter orthosis (Figure 1 middle), the moment of force to prevent fall 
acts on the subject to provide stability. Therefore, although foot sole plates are not currently used to prevent falls, they can be used for this purpose by controlling the plantar height.

A magnetorheological (MR) fluid brake, which is compact, light, and power-saving, but is able to generate a large amount of force and is highly responsive, is used to control the plantar height only when there is a risk of falling. The MR fluid is a fluid in which micron-sized magnetic particles are dispersed in the carrier fluid (Rabinow, 1948). The yield shear stress changes in a few milliseconds depending on the external magnetic field. MR fluid has been applied to devices such as dampers and engine mounts for vibration control by taking advantage of its force control range and responsiveness (Shiraishi, Miida, et al., 2011; Shiraishi, Misaki, 2016; Phu, Choi, 2019; Phu, Choi, et al., 2014; Phu, Shah, et al., 2014). The MR fluid response to the magnetic field can be applied to a device to generate a frictional force against an external force, which can be used as a braking force. This study aims to develop an orthosis with a controllable plantar height to prevent fall of people with walking disabilities using a compact MR fluid brake. First, we designed and fabricated a compact MR fluid brake that can maintain the plantar height. Next, we combined it with footwear, a controller, batteries, and a sensor to develop an orthosis for fall prevention. Subsequently, the MR fluid brake characteristics, including the component of the generated force and its magnitude and the response to the impact load by a human walking, were examined to verify whether the MR fluid brake could act as a brake that can control the plantar height. Finally, an experiment was conducted to evaluate the fall prevention effect of the orthosis.

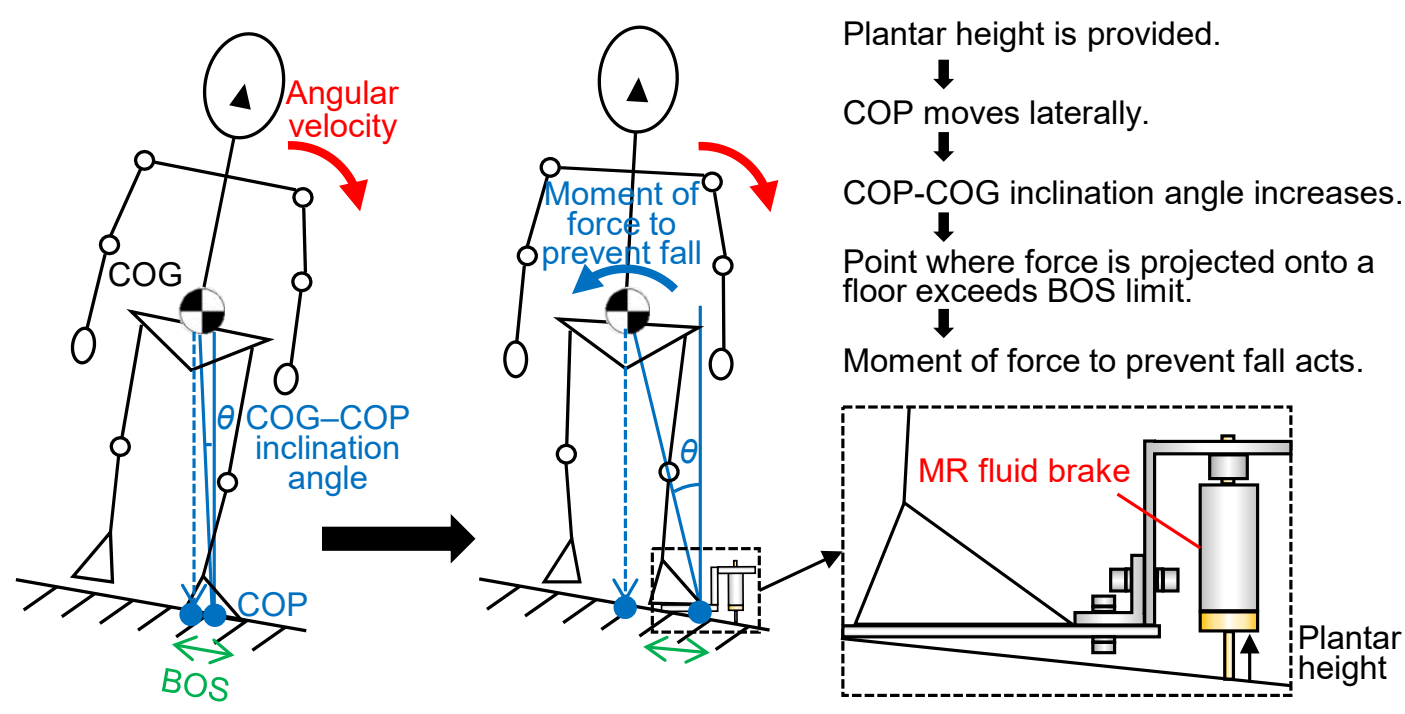

Figure 1 Image of fall prevention with the MR fluid brake. The COP moves laterally, and the COG-COP inclination angle increases by giving the plantar height. The COG-COP inclination angle is a gait stability index.

\section{Orthosis with a controllable plantar height for fall prevention}

\subsection{Design of a compact magnetorheological fluid brake}


The MR fluid brake was designed to be compact and light, able to generate enough force to support body weight, and is responsive enough to prevent falls. The MR fluid brake structure mainly consisted of a cylinder and a piston (Figure 2). An O-ring between the piston and the cylinder was unnecessary to reduce the sliding resistance. The magnetic circuit spanned both the piston and the cylinder. A double-rod type, which did not require air to interpolate the volume of the piston rod, was adopted to prevent air from entering the MR fluid and the which would prevent the device from acting as a brake. Although the mass that affects the walking speed and the muscle activity is $1.5 \mathrm{~kg}$ (Omuro, Shiba, et al., 2011), the maximum weight of the device should be approximately $300 \mathrm{~g}$ based on the experience of physical therapists observing patients walking. The MR fluid brake stroke was set at $7 \mathrm{~mm}$ to allow a margin for the Iritani plantar plate, which uses a 6 mm-thick arch pad (Iritani, 2017).

Figure 3 illustrates the design flow. The generated force of the MR fluid device, in which the pressure mode is dominant, was obtained by the product of the pressure-receiving area of the piston and the pressure difference before and after the orifice. We determined the pressure difference and pressure-receiving area after determining the desired withstand load. To achieve a compact MR fluid brake, the pressure-receiving area must be small; therefore, the pressure difference must be increased. Since the channel height must be reduced to achieve a large pressure difference, it was set as $0.5 \mathrm{~mm}$ as that is minimum setting from the general in the range of 0.5 to $2 \mathrm{~mm}$ (Nanthakumar and Jancirani, 2019). The number of coil turns and piston outer diameter were determined by $B-H$ curve of the MR fluid and the experimentally-derived equation as described below, respectively.

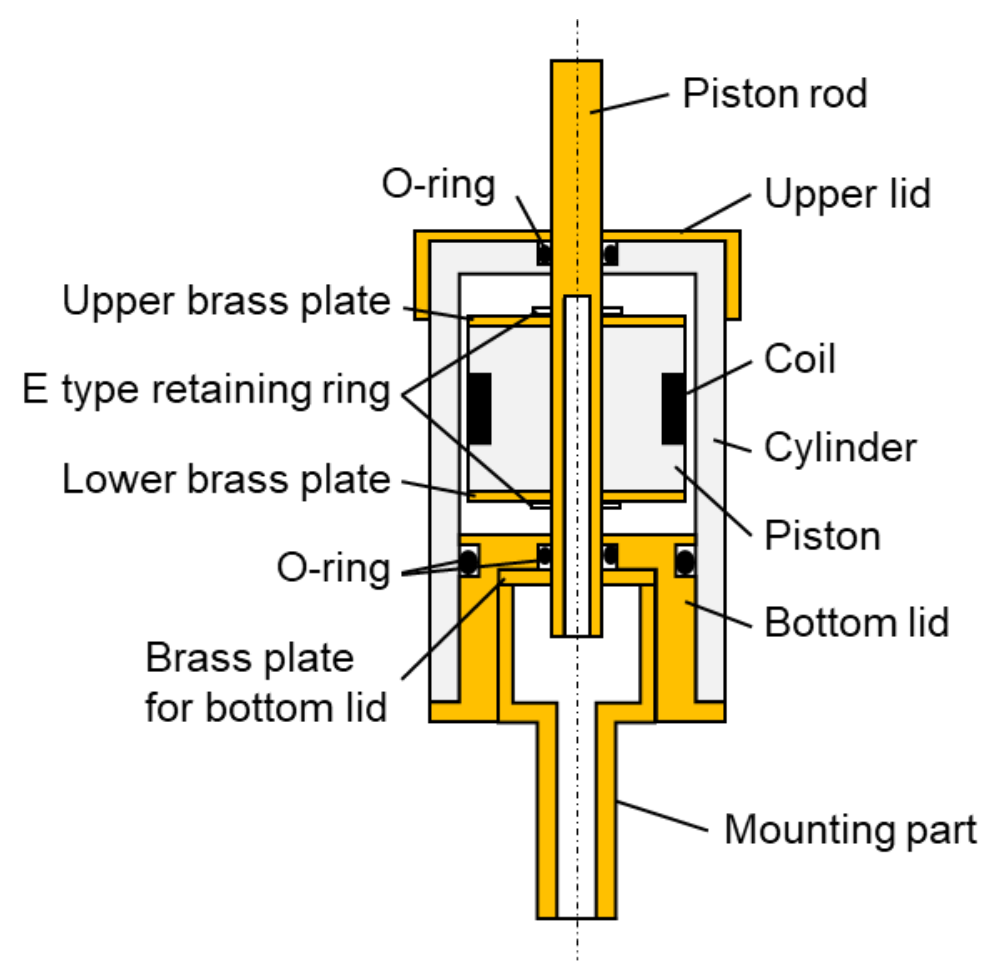

Figure 2 Schematic diagram of the MR fluid brake. 


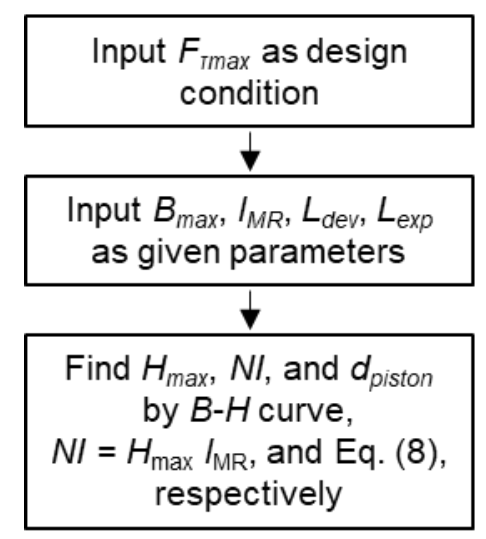

$F_{\text {Imax }}:$ Withstand load

$B_{\max }$ : Maximum operating magnetic flux density

$I_{M R}$ : Channel height

(Minimum value among commonly used size)

$L_{\text {dev }}$ : Flow path length, where the magnetic field is applied in MR fluid brake to be designed

$L_{\text {exp }}$ : Flow path length, where the magnetic field is applied in the characterization device

$H_{\text {max }}$ : Maximum magnetic field strength

$N$ : Number of coil turns

I: Current

$d_{\text {piston: }}$ Piston outer diameter

Figure 3 Design process flowchart.

\section{Withstand load}

Equation (1) expresses the force $F$ generated by the MR fluid brake.

$$
F=F_{\tau}+F_{\eta}+F_{f}
$$

where, $F_{\tau}$ : force due to the yield stress of the MR fluid; $F_{\eta}$ : force due to viscosity as a Newtonian fluid; and $F_{f}$ : sliding friction force by the seal material.

Of these forces, $F_{\tau}$ is the force that can be controlled by an external magnetic field, while $F_{\eta}$ and $F_{f}$ are the forces that occur, even when no magnetic field is applied. $F_{\tau}$ and $F_{f}$ act as the braking force. The MR fluid brake was placed outside of the foot, and the stepping force was transmitted to the MR fluid brake using a load-bearing part based on the principle of leverage. Based on the foot width and the MR fluid brake position, the distance from the center of rotation to the load-bearing target point was determined as $40 \mathrm{~mm}$, and the distance to the MR fluid brake was $90 \mathrm{~mm}$ (Figure 4).

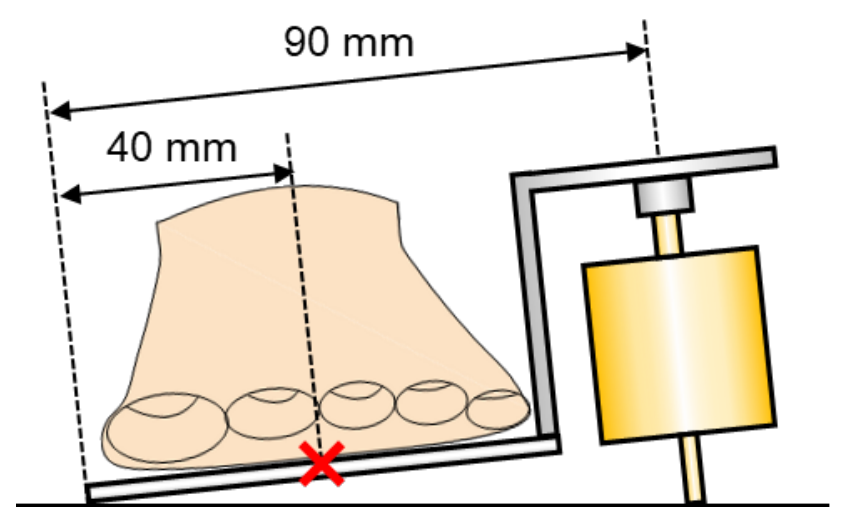


We assumed that the entire body weight was applied to the load-bearing part. It is reported that the heaviest average weight for men was $70.3 \mathrm{~kg}$ for 40 to 49 years old, while that for women was $55.2 \mathrm{~kg}$ for 50 to 59 years old (Statistic Bureau of Japan, 2016). Assuming that the target heaviest weight was $70.3 \mathrm{~kg}$, the load applied to the MR fluid brake can be calculated as follows:

$$
70.3[\mathrm{~kg}] \times 9.8\left[\mathrm{~m} / \mathrm{s}^{2}\right] \times \frac{40[\mathrm{~mm}]}{90[\mathrm{~mm}]}=306.1[\mathrm{~N}]
$$

Assuming that the safety factor of disc brakes is 1.5 for reference (Naikwadi, 2017), the allowable load is as follows:

$$
306.1[\mathrm{~N}] \times 1.5=459.1[\mathrm{~N}]
$$

The influence of $F_{\eta}$ and $F_{f}$ was smaller than that of $F_{\tau}$ when the electric current was sufficiently large. Therefore, the maximum controllable force of the MR fluid brake $F_{\tau \max }$ was set as the allowable load, and $F_{\tau \max }=460 \mathrm{~N}$.

\section{Piston}

Among the many components, the piston design is described because this part is the key to reducing the size and weight of the MR fluid brake. The material is structural carbon steel commonly used in DC electromagnets. Among structural carbon steels, S10C was used because of its lower carbon content, which has been found to give better magnetic properties (Ishiguro, Tsuboshima, et al., 1978). Considering the length of the MR fluid brake when placed outside of the foot, the piston height $h_{\text {piston }}$ was determined as $12.5 \mathrm{~mm}$. The cross-sectional area perpendicular to magnetic fluxes I, II, and III should be designed to be the same to avoid magnetic saturation due to a local decrease in the cross-sectional area in a magnetic circuit (Figure 5). The piston outer diameter $d_{\text {piston }}$ was determined using the equation experimentally derived under the condition that cross-sectional areas I and II are the same. 


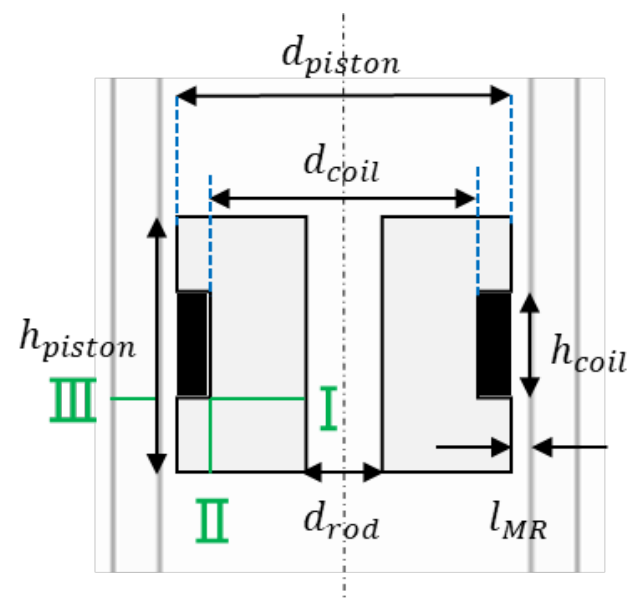

Figure 5 Detailed view of the piston.

$$
h_{\text {piston }}-h_{\text {coil }}=\frac{d_{\text {coil }}^{2}-d_{\text {rod }}^{2}}{2 d_{\text {coil }}}
$$

As a fundamental property of the MR fluid in the pressure mode, Equation (5) was experimentally derived for the relation between the pressure difference before and after the orifice and the magnetic flux density (Shiraishi and Morishita, 2004).

$$
\Delta p=\left(6.89 \times 10^{3} / l_{M R}\right) B^{2}+b
$$

The value of $b$ does not significantly change with the change of the channel height; hence, we set $b=1.37 \times 10^{5}$, which is the value at $l_{M R}=0.5 \mathrm{~mm}$. The force $F$ generated by the MR fluid device, where the pressure mode is dominant, can be obtained from the product of the pressure-receiving area $A$ of the piston and the pressure difference $\Delta p$ before and after the orifice. The pressure difference is divided into a term that depends on the magnetic flux density generated in the MR fluid by applying an external magnetic field and a term that does not depend on the magnetic flux density. The maximum force $F_{\text {tmax }}$ that can be controlled by the MR fluid brake is obtained by increasing the pressure difference to $\Delta p_{\tau \max }$ by an external magnetic field:

$$
F_{\text {tmax }}=\Delta p_{\text {tmax }} A
$$

$F_{\text {tmax }}$ is proportional to the channel length in the section where the magnetic field is applied; therefore, it can be expressed as follows from Equations (5) and (6). 


$$
F_{\tau \max }=\left(\frac{6.89 \times 10^{3}}{l_{M R}} B_{\max }^{2}+b\right) A L_{d e v} / L_{\exp }
$$

Equation (8) can be derived by solving for $l_{M R}$ and substituting Equation (4).

$$
l_{M R}=6.89 \times 10^{3} B_{\text {max }}^{2}\left(\frac{F_{\tau \text { max }}}{\frac{\pi d_{\text {piston }}^{2}}{4}-\frac{\pi d_{\text {rod }}^{2}}{4}} \frac{2 d_{\text {coil }} L_{\text {exp }}}{d_{\text {coil }}^{2}-d_{\text {rod }}^{2}}-b\right)^{-1}
$$

$L_{\text {exp }}$ is the length of the flow path, where the magnetic field is applied in the characterization device (i.e., $20 \mathrm{~mm}$ ) (Shiraishi and Morishita, 2004). The piston rod outer diameter $d_{\text {rod }}$ was set to $5 \mathrm{~mm}$ for a small size. The dent for

winding the coil $\frac{\left(d_{\text {piston }}-d_{\text {coil }}\right)}{2}$ was determined by considering the copper wire diameter and the number of coil layers. If the coil winding dent is known, the inner diameter of the coil $d_{\text {coil }}$ can be determined by the piston outer diameter $d_{\text {piston }}$; therefore, Equation (8) becomes a relation between $l_{M R}$ and $d_{\text {piston }}$ when $B_{\max }$ is determined. We set the maximum operating magnetic flux density $B_{\max }$ to $0.9 \mathrm{~T}$. From the above, the channel height and piston volume are calculated when the piston outer diameter is varied as a parameter. The smaller the piston outer diameter, the smaller the piston volume, which leads to a smaller overall device; however, a smaller channel height is required to generate the desired force. Assuming $l_{M R}=0.5 \mathrm{~mm}$ to prevent clogging, the piston outer diameter is set to $15.5 \mathrm{~mm}$.

\section{Responsiveness}

As a control method, when the MR fluid brake was incorporated into a fall prevention device, two AA batteries were used to supply electric current to the MR fluid brake when the lateral inclination of the road exceeded a certain angle. However, the self-inductance of the coil acted to cancel the current; thus, it took time for the current to rise. The time constant $\tau$ was calculated to evaluate whether the device can prevent fall in time. The time constant can be calculated as the self-inductance $L$ and resistance $R$ from Equation (9).

$$
\tau=\frac{L}{R}
$$

The self-inductance is obtained from the number of coil turns that satisfy the desired magnetic flux density and the coil height obtained earlier. The time constant is $179 \mathrm{~ms}$.

We then consider whether this time constant is sufficient to prevent falling. In this study, an electric current must be applied when a lateral inclination of the walking path is detected by an accelerometer attached to the target foot 
before the next initial contact of the target foot. Considering the gait cycle from the initial ground contact of the target foot $(0 \%)$ to the next initial ground contact $(100 \%)$, the lateral inclination should be detected at the foot flat phase (10\%); the electric current should start to flow after the toe off $(60 \%)$; and the MR fluid brake should prepare to generate force against the external force by the next initial ground contact (100\%) described in control low of Section 2.2 in detail (Figure 6). Since a walking cycle is approximately $1 \mathrm{~s}$ (Murray, Kory, et al., 1969), the time from the start of the current flow to the response of the MR fluid must be within $400 \mathrm{~ms}$ or $40 \%$ of a walking cycle considering applying the electric current at the toe off. Assuming that the sampling period of the sensor is $10 \mathrm{~ms}$, the required response time of the MR fluid is $400 \mathrm{~ms}-179 \mathrm{~ms}-10 \mathrm{~ms}=211 \mathrm{~ms}$. This MR fluid brake can be used to prevent fall because its response time is a few milliseconds.

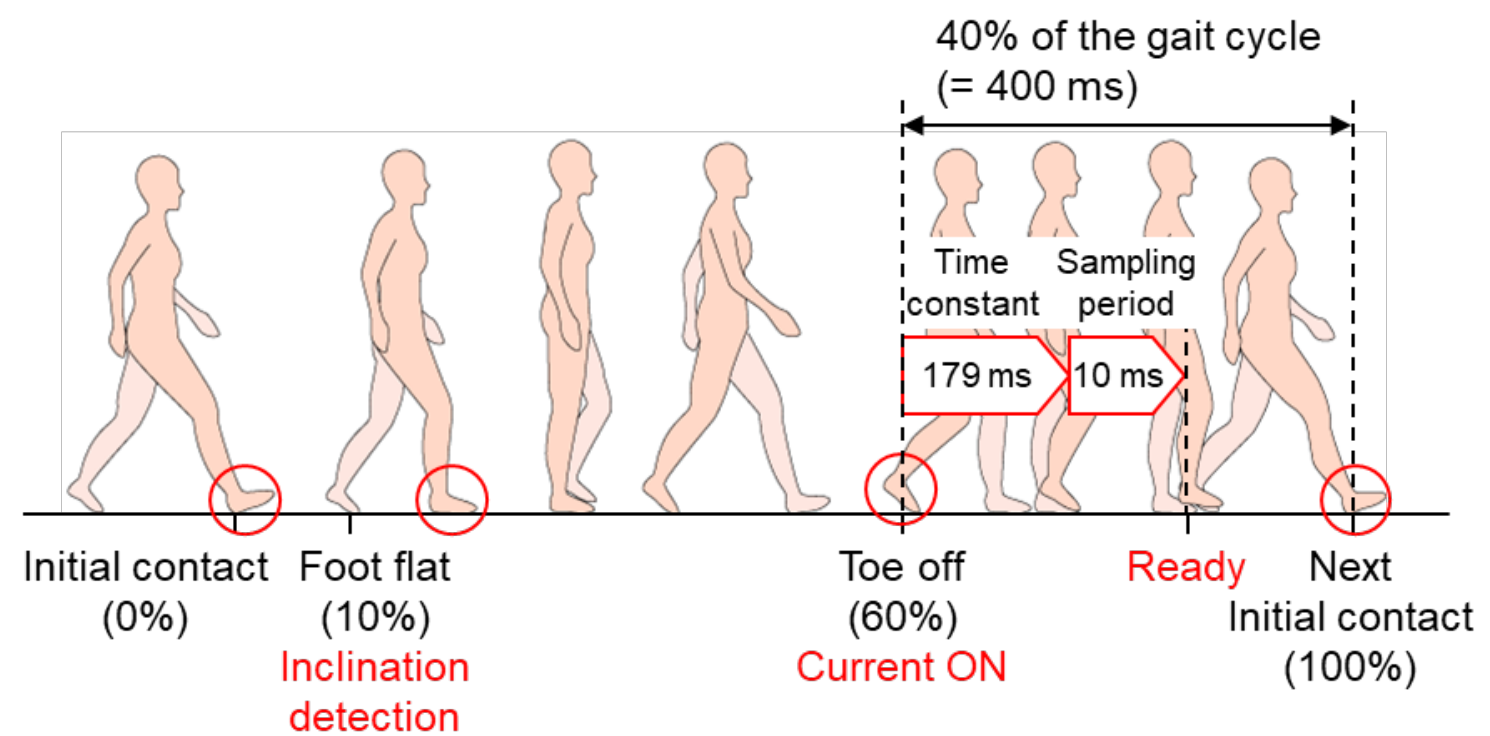

Figure 6 Schematic diagram of walking cycle (Andrew, Arima, et al., 2018) adding inclination detection and current outputting timing for the fall prevention control.

\section{Parameter}

We designed and fabricated an MR fluid brake that is compact, light, and power-saving, generates enough force to support the body weight, and is responsive enough to prevent falls (Figure 7). Table 1 lists the parameters determined in the design. 

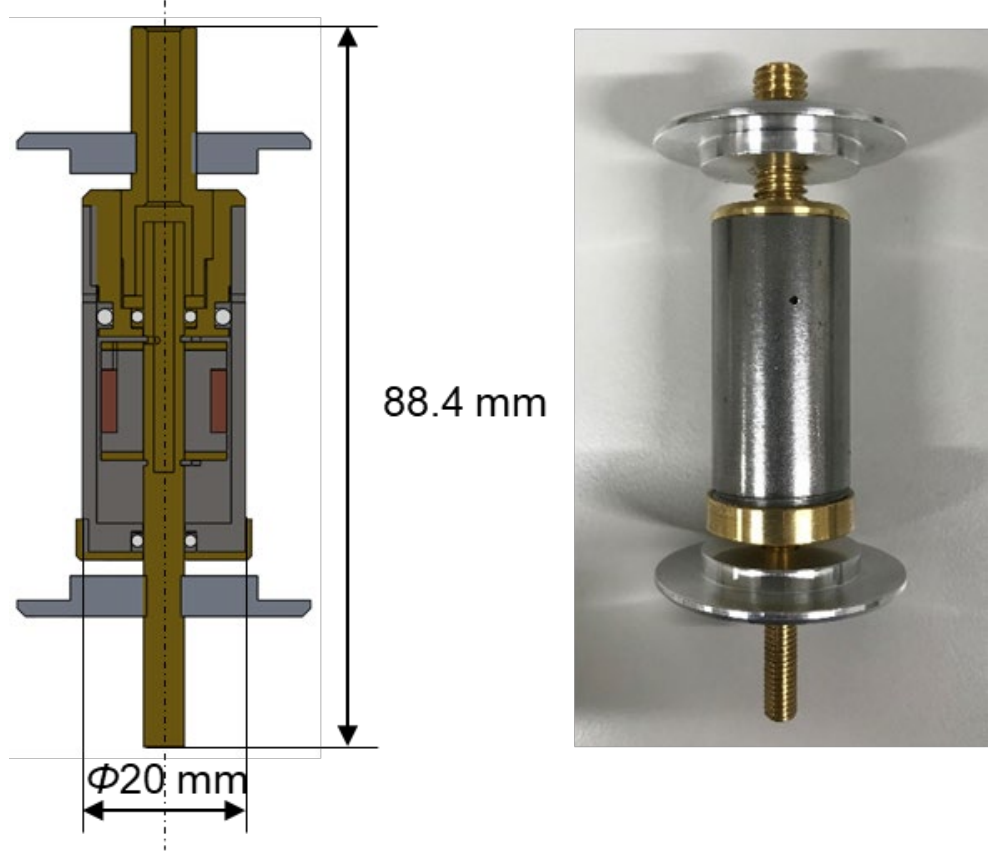

Figure 7 Compact and light MR fluid brake $(128 \mathrm{~g})$.

Table 1 Parameters of the MR fluid brake.

\begin{tabular}{|l|r|}
\hline \multicolumn{1}{|c|}{ Parameter } & \multicolumn{1}{c|}{ Value } \\
\hline Mass & $128 \mathrm{~g}$ \\
\hline Piston outer diameter $\left(d_{\text {piston }}\right)$ & $15.5 \mathrm{~mm}$ \\
\hline Cylinder outer diameter $\left(d_{\text {cylinder }}\right)$ & $20 \mathrm{~mm}$ \\
\hline Channel height $\left(l_{\text {MR }}\right)$ & $0.5 \mathrm{~mm}$ \\
\hline Coil height $\left(h_{\text {coil }}\right)$ & $7.7 \mathrm{~mm}$ \\
\hline Stroke & $7 \mathrm{~mm}$ \\
\hline Number of coil turns $(N)$ & 80 \\
\hline Time constant $(\tau)$ & $179 \mathrm{~ms}$ \\
\hline
\end{tabular}

\subsection{Orthosis development}

The MR fluid brake was combined with sandals, a controller, batteries, and a sensor to develop a fall prevention device (Figure 8). Sandals with a stiff sole were chosen such that the MR fluid brake effect on the plantar height can be easily seen. As for the point where the adjustment to the plantar height is applied, the reaction when the plantar height is given to the entire sole differs from person to person, which may make the gait unstable. Dynamic balance is improved by supporting the cuboid bone (Takata, Takeda, et al., 2016); hence, the plantar height is given not to the entire sole, but to the vicinity of the tuberosity of the fifth metatarsal bone, including the cuboid bone. The MR fluid brake was placed outside of the sandal, and the stepping force was transmitted to the MR fluid brake by using a loadbearing part based on the leverage principle. An inertial measurement unit (IMU, Umemoto LLC, REES-01) that detects three-axis acceleration and three-axis angular velocity was used to measure the road inclination angle and 
detect the ground contact. The sensor was controlled by a microcontroller (Arduino, Arduino Uno Rev3 A000066). The controller and the IMU location were placed above the ankle so as not interfere with walking, if they were placed as close to the ankle joint as possible. However, their mass was relatively light (approximately $40 \mathrm{~g}$ ). The IMU was used to determine the posture from the acceleration of the gravity component when the foot stopped moving momentarily after the foot made contact with the ground; thus, we attached the sensor directly to the belt part of the instep, which showed little relative motion with the foot. Two batteries were required: one to supply power to the controller and the other for the MR fluid brake. Both batteries were mounted on the lower leg. For the controller, we chose a battery that could continuously supply power, even at low currents without activating the protection function. The MR fluid brake required $1.3 \mathrm{~V}$; hence, we used two AA batteries of $1.5 \mathrm{~V}$ connected in series to provide sufficient power. Since the voltage is $3 \mathrm{~V}$ and the resistance is $0.89 \Omega$, the input current is $3.4 \mathrm{~A}$ which is enough for the MR fluid brake to act as a brake as described below.

(a)
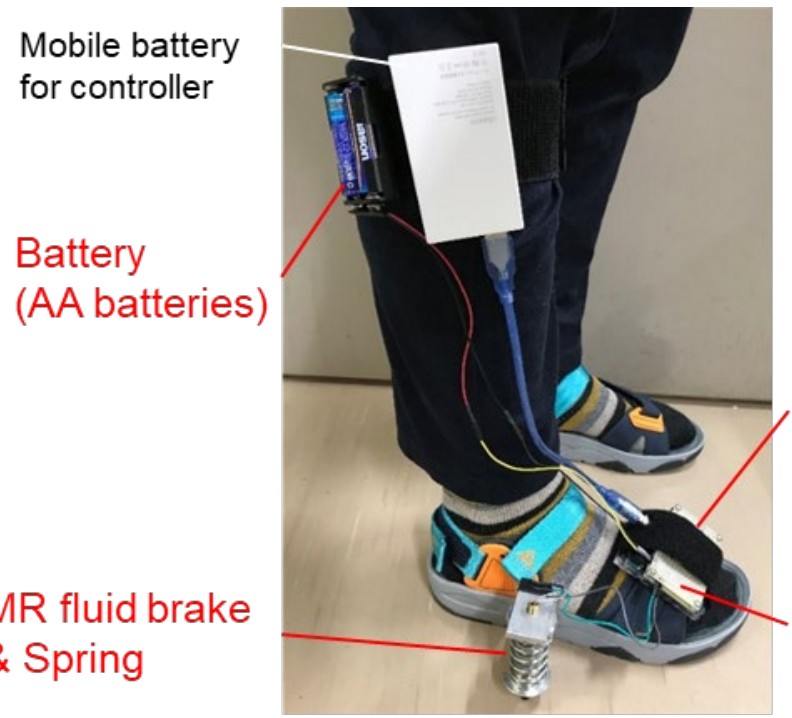

Controller

(Arduino Uno Rev3)

Inertial

measurement

unit

(b)

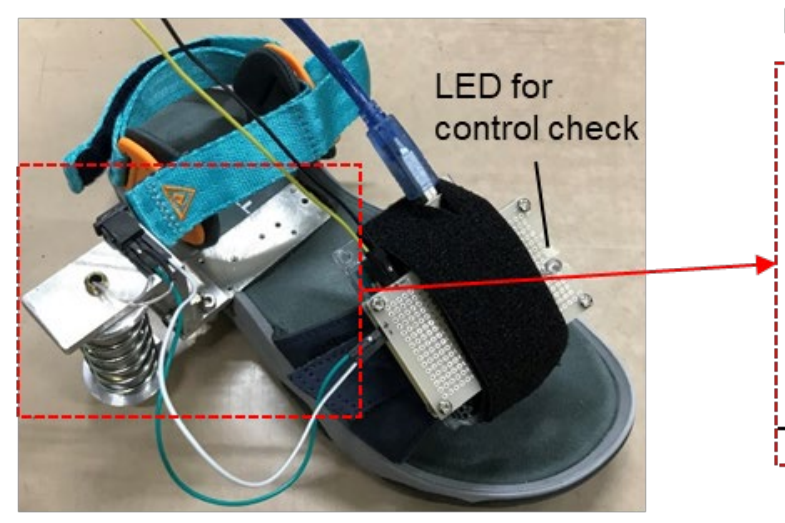

MR fluid brake

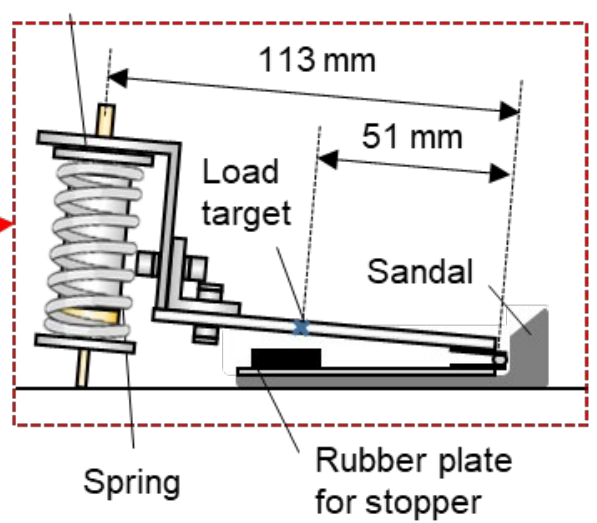

Figure 8 Fall prevention orthosis using the MR fluid brake: (a) Overall view of the fall prevention orthosis using MR fluid brake. (b) Enlarged view near the sandal. The MR fluid brake is placed outside of the sandal. 


\section{Control law}

When the lateral inclination angle of the road exceeds $5^{\circ}$ during walking, the switch is turned ON 0.5 seconds after the angle exceeds $5^{\circ}$ and turned OFF 1 second later so that the electric current flows at the next foot contact. According to Road Structure Ordinance, the standard cross slope of a sidewalk is $2 \%\left(1.1^{\circ}\right)$ or less, but the slope where a vehicle enters a sidewalk is $15 \%\left(8.5^{\circ}\right)$ or less (MLIT, 2003). The threshold was set at $5^{\circ}$ considering fall prevention when people with balance disorders walk on a slope greater than the standard cross slope. We used the magnitude of the acceleration of gravity distributed to each axis by the tilt of the IMU itself for lateral inclination angle measurement. The orientation of the axes of the IMU attached to the sandal is shown in Figure 9. The acceleration of each axis is $a_{x}, a_{y}$, or $a_{z}$. The angular velocity around each axis is $\omega_{x}, \omega_{y}$, or $\omega_{z}$. The lateral inclination angle is calculated from Equation (10).

$$
\theta_{x}=\tan ^{-1} \frac{a_{y}}{\sqrt{a_{x}^{2}+a_{z}^{2}}}
$$

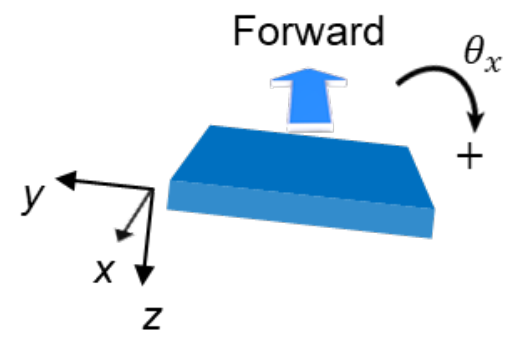

Figure 9 Axis of the IMU attached to a sandal. A positive roll angle indicates clockwise motion.

However, the values observed by the sensor are a mixture of those caused by the foot placed and those caused by the lateral inclination angle of the road. Therefore, to measure the lateral inclination angle, it is necessary to recognize the state of foot contact where the foot stops moving temporarily. In a preliminary experiment, the IMU was attached to the toe, and acceleration and angular velocity were measured during walking. As a result, when the difference between the composite acceleration $a$ and the acceleration of gravity $g$ is less than a certain small value $\Delta a$ and the composite angular velocity $\omega$ is less than a certain small value $\Delta \omega$ for a certain period of time $\Delta t$, the state is considered to be foot contact. In Equation (11), $s_{\text {con }}$ is a variable representing the foot contact state (1: foot contact, 0 : otherwise) in walking and depends on time $t$.

$$
s_{\text {con }}=\left\{\begin{array}{c}
1, \text { if }|a|-g<\Delta a \text { and }|\omega|<\Delta \omega \text { during } t-\Delta t \sim t \\
0, \text { otherwise }
\end{array}\right.
$$

where , $g=9.8\left[\mathrm{~m} / \mathrm{s}^{2}\right], \Delta a=1.96\left[\mathrm{~m} / \mathrm{s}^{2}\right],|\omega|=\sqrt{\omega_{x}^{2}+\omega_{y}^{2}+\omega_{z}^{2}}, \Delta \omega=25[\% / \mathrm{s}], \Delta t=0.1[\mathrm{~s}]$ 
From the above, the control current $I$ is expressed as Equation (12) in the case of a time in a walking cycle $T$.

$$
I=\left\{\begin{array}{c}
I_{0} \text { during } t+T / 2 \sim t+3 T / 2, \text { if } \theta_{x}>5^{\circ} \text { and } s_{\text {con }}=1 \\
0, \text { otherwise }
\end{array}\right.
$$

where $I_{0}=3.4[\mathrm{~A}], T=1[\mathrm{~s}]$.

We verified that the control law worked correctly by walking on a $10^{\circ}$ inclined surface. A slope was placed in the middle of a flat surface, and a subject wearing a fall prevention device walked along the path (Figure 10). After the right foot with the MR fluid brake makes contacts with the ground on the slope, and when the MR fluid brake is released, it is set to $0 \mathrm{~s}$. The time when the current started to flow $\left(t_{\text {current_ON }}\right)$, the time when the second step of the right foot made contact with the ground ( $\left.t_{\text {initial_contact }}\right)$, the time when the MR fluid brake was released on the second step of the right foot $\left(t_{\text {release }}\right)$, and the time when the current stopped ( $\left.t_{\text {current_OFF }}\right)$ were measured. These trials were performed ten times. The sampling frequency was $30 \mathrm{~Hz}$. The results showed that the current started to flow between the MR fluid brake release of the first step of the right foot and the contact of the second step of the right foot, and the current stopped after the MR fluid brake release of the second step of the right foot in all ten cases (Table 2). Therefore, it was confirmed that the control always operated correctly. 
(a)

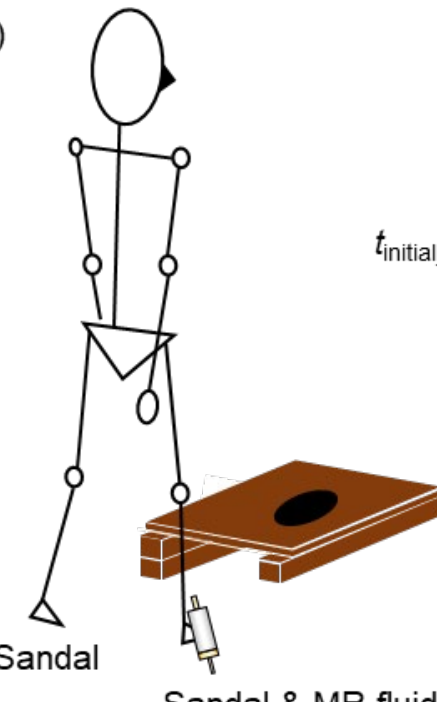

Sandal \& MR fluid brake

\section{$t_{\text {current_OFF }}$ : Current stops to flow}

$t_{\text {release }}:$ MR fluid brake releases

initial_contact: Right foot contacts
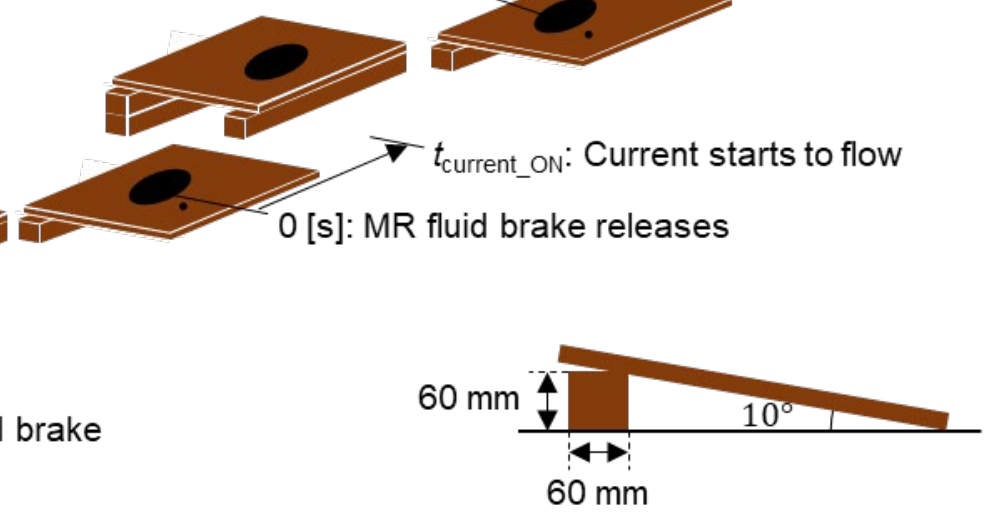

(b)

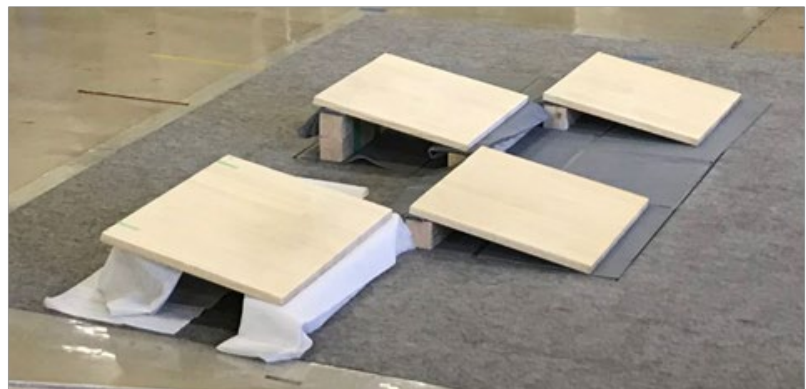

Figure 10

The experimental setup for verification of sensor operation. (a) Schematic and (b) photograph.

Table 2 Checking the control of fall prevention device.

\begin{tabular}{|r|r|r|r|r|}
\hline Trial No. & $\boldsymbol{t}_{\text {current_ON [s] }}$ & $\boldsymbol{t}_{\text {initial_contact [s] }}$ & $\boldsymbol{t}_{\text {release [s] }}$ & $\boldsymbol{t}_{\text {current_OFF [s] }}$ \\
\hline 1 & 0.2 & 0.67 & 1.24 & 2.44 \\
\hline 2 & 0.17 & 0.6 & 1.23 & 2.4 \\
\hline 3 & 0.27 & 0.6 & 1.24 & 2.4 \\
\hline 4 & 0.2 & 0.6 & 1.23 & 2.4 \\
\hline 5 & 0.2 & 0.6 & 1.27 & 2.4 \\
\hline 6 & 0.23 & 0.6 & 1.23 & 2.4 \\
\hline 7 & 0.2 & 0.6 & 1.27 & 2.4 \\
\hline 8 & 0.23 & 0.57 & 1.2 & 2.37 \\
\hline 9 & 0.2 & 0.6 & 1.23 & 2.4 \\
\hline 10 & 0.24 & 0.57 & 1.2 & 2.37 \\
\hline
\end{tabular}

\section{Performance test of the MR fluid brake}

3.1 Hysteresis loops for the MR fluid brake 
We verified that the generated force was small enough not to act as a brake during normal walking. We also verified the frictional force necessary to act as a brake was dominant when there was a risk of falling and that the device performance was sufficient for use as a fall prevention device.

\section{Methods}

The MR fluid brake was fixed horizontally to the floor, and a spherical plain bearing was used at the connection between the piston rod and the excitation part (Figure 11). The reason for installing this part was to prevent the MR fluid brake from being damaged by excessive force when the direction of vibration and the MR fluid brake axis were not aligned. A jig was used to prevent the excitation part from rotating during vibration, which would loosen the upper lid and cause a leak of MR fluid. The piston displacement and the force generated by the MR fluid brake were measured by a displacement sensor and a load cell attached to the hydraulic shaker and recorded by an oscilloscope. A bipolar power supply was used as the power source to supply electric current to the coils in the MR fluid brake. A hydraulic shaker was used to apply a sinusoidal forced displacement to the MR fluid brake, and the generated force and the piston displacement were measured. The experimental conditions were amplitude of $2.5 \mathrm{~mm}$, vibration frequency of $1 \mathrm{~Hz}$, and electric current of $0,0.4,0.8,1.2$, and $1.5 \mathrm{~A}$. 
(a)

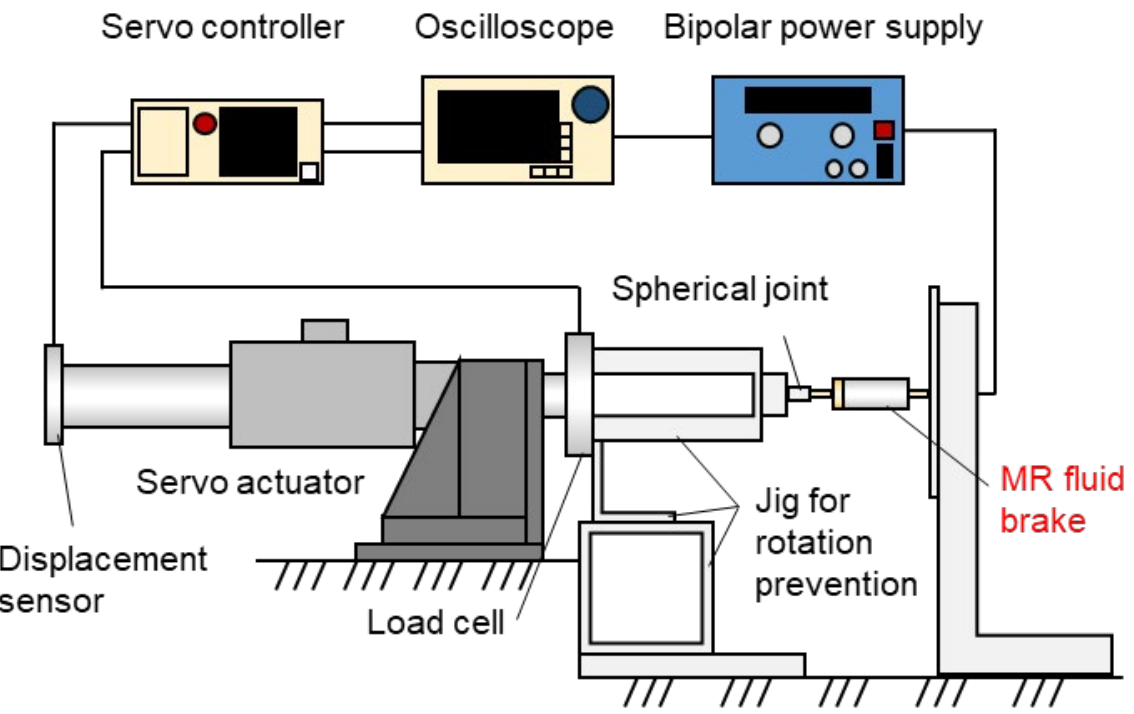

(b)

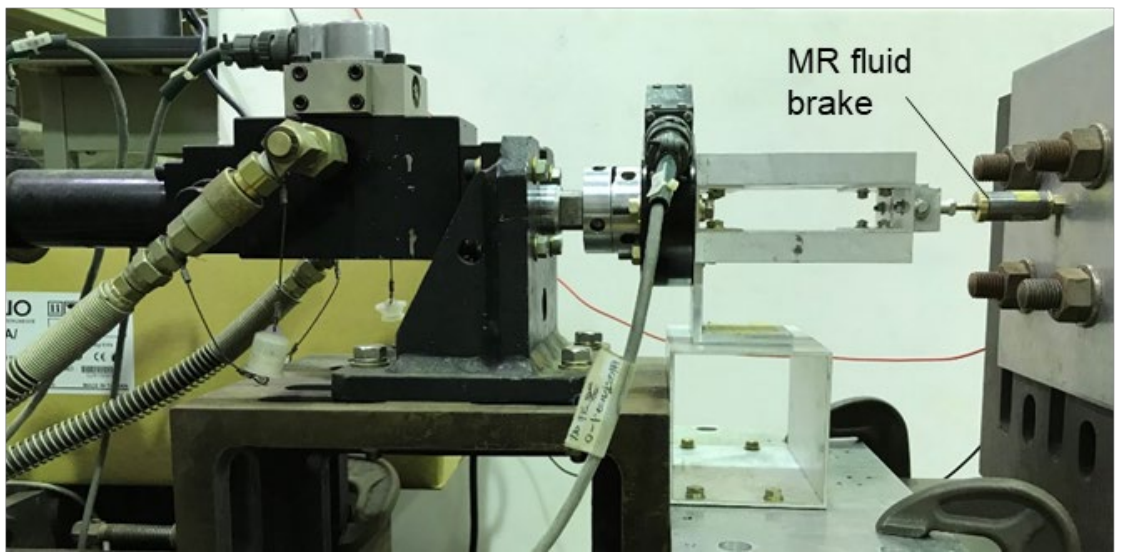

Figure 11 The experimental setup for vibration test. (a) Schematic and (b) photograph.

\section{Results and discussion}

The hysteresis loops of the piston displacement and the generated force were almost rectangular representing the frictional force required to act as a brake, with and the force increasing as the current increased (Figure 12). The maximum force at $0 \mathrm{~A}$ was approximately $17 \mathrm{~N}$, which was as small as expected. Compared to the MR fluid brake developed in the previous study, which had a maximum force of approximately $180 \mathrm{~N}$ at $0 \mathrm{~A}$ (Morimura, Yamamoto, et al., 2019), the present design was confirmed to have a smaller initial resistance. In a preliminary experiment, we fabricated a slipper with an MR fluid brake that gives plantar height vicinity of the tuberosity of the fifth metatarsal and examined the force applied to the MR fluid brake when a person applied a load. Assuming an average weight of $70.3 \mathrm{~kg}$ used in the design, approximately $69 \mathrm{~N}$ was applied to the MR fluid brake, therefore, the force of $17 \mathrm{~N}$ was small enough not to act as a brake. The maximum force was approximately $125 \mathrm{~N}$. Assuming that $69 \mathrm{~N}$ was applied to the MR fluid brake from the preliminary experiment as before, $125 \mathrm{~N}$ was large enough to act as a brake.

In the MR fluid brake developed in the previous study, the force was not generated immediately after the piston 
motion direction changed due to the effect of compressibility caused by air in the MR fluid (Morimura, Yamamoto, et al., 2019). On the other hand, the shape of the hysteresis loops was not perfectly rectangular in this experiment, but the force rose immediately after the direction of motion changed. The displacement required for the force to rise was approximately $0.5 \mathrm{~mm}$ at $1.5 \mathrm{~A}$, but this was not a problem because the plantar height commonly used in physical therapy is a few millimeters to $6 \mathrm{~mm}$ (Iritani, 2017), and the displacement of $0.5 \mathrm{~mm}$ is smaller than this height. Therefore, it was confirmed that this device satisfied the performance requirements for a brake.

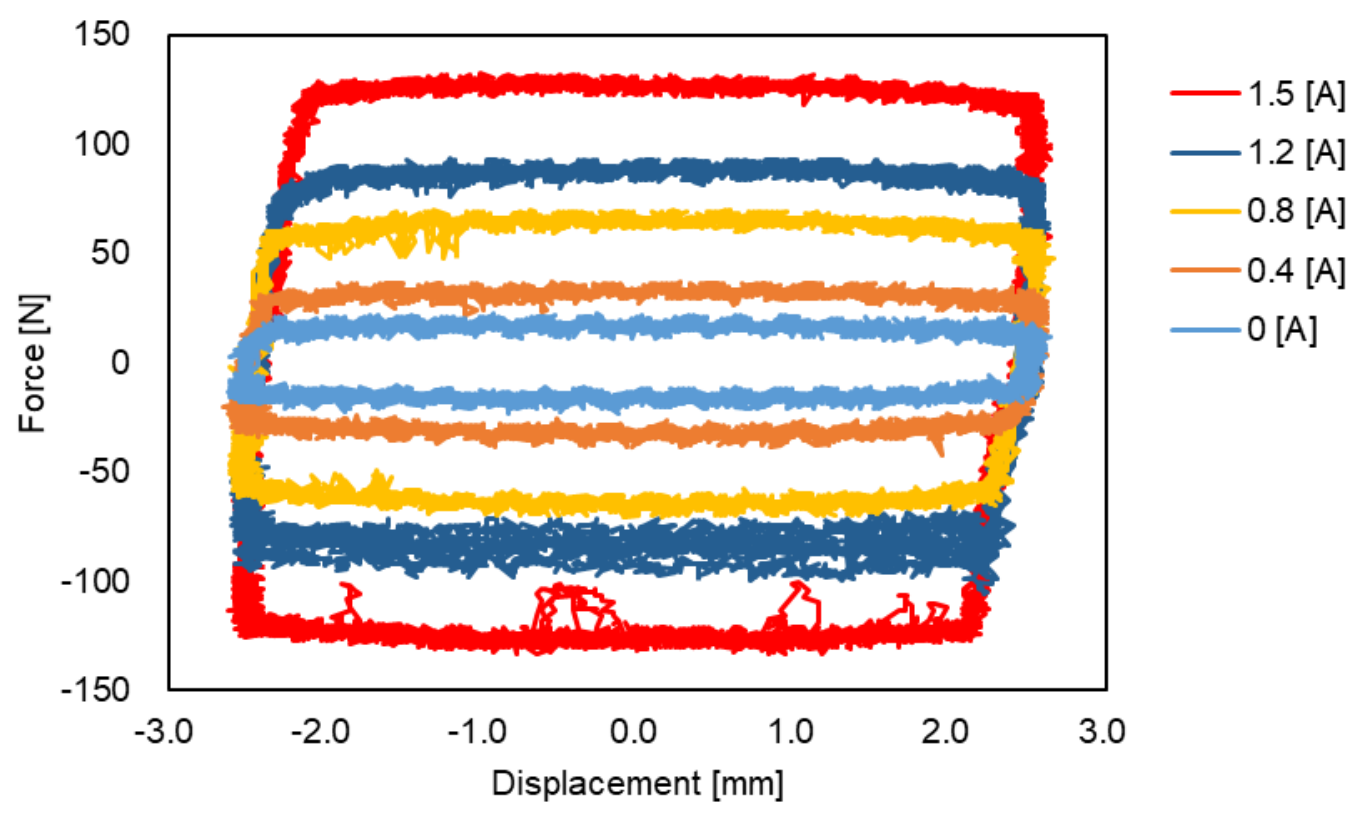

Figure 12 Vibration test results of MR fluid brake with an amplitude of $2.5 \mathrm{~mm}$ and a frequency of $1 \mathrm{~Hz}$.

\subsection{Response characteristics of the MR fluid brake to the impact load given by human walking}

In an actual application, the MR fluid brake is attached to a shoe. A load is applied to the MR fluid brake every time the shoe makes contact with the ground during walking. If there is a risk of falling, the piston is maintained constantly against the load by the current from an external power source. The load applied by a human is not constant, but dynamic, including the impact load; hence, we applied load under the expected conditions of use to the MR fluid brake and verified whether it operated as a brake when an electric current was applied. The piston was displaced against external forces when no electric current was applied, such that the MR fluid brake did not give the plantar height, except when there was a risk of falling.

\section{Methods}

The MR fluid brake was attached to a slipper and fixed to the ground to apply a load under actual use (Figure 
13). The slipper height was adjusted so that the load-bearing part would be horizontal when the piston rod was most retracted. The upper part of the slipper was removed such that the foot could contact the top of the slipper. When the slipper was stepped on with the foot, load was applied to the load-bearing part attached to the slipper. Subsequently, load was applied to the MR fluid brake. The load-bearing part was large enough to give the plantar height at the tuberosity of the fifth metatarsal. The load applied to the MR fluid brake and the piston displacement were measured by a load cell and a laser displacement sensor, respectively, and recorded by an oscilloscope. Even if the piston rod is extended by a maximum of $7 \mathrm{~mm}$, the misalignment between the MR fluid brake axis and the vertical axis is so small that the experimental setup has sufficient precision to measure $99.8 \%$ of the original force. A bipolar power supply was used to supply the electric current to the coils in the MR fluid brake. We started the experiment with the right foot one step behind, then swung the right foot forward and contacted the ground directly on the slipper. The experiment was completed when the toes left the slipper. The load applied to the MR fluid brake and the piston displacement were measured. The subject was a healthy person aging 23 years and weighing $55 \mathrm{~kg}$. Two conditions were tested: 0 A and 1.5 A current.
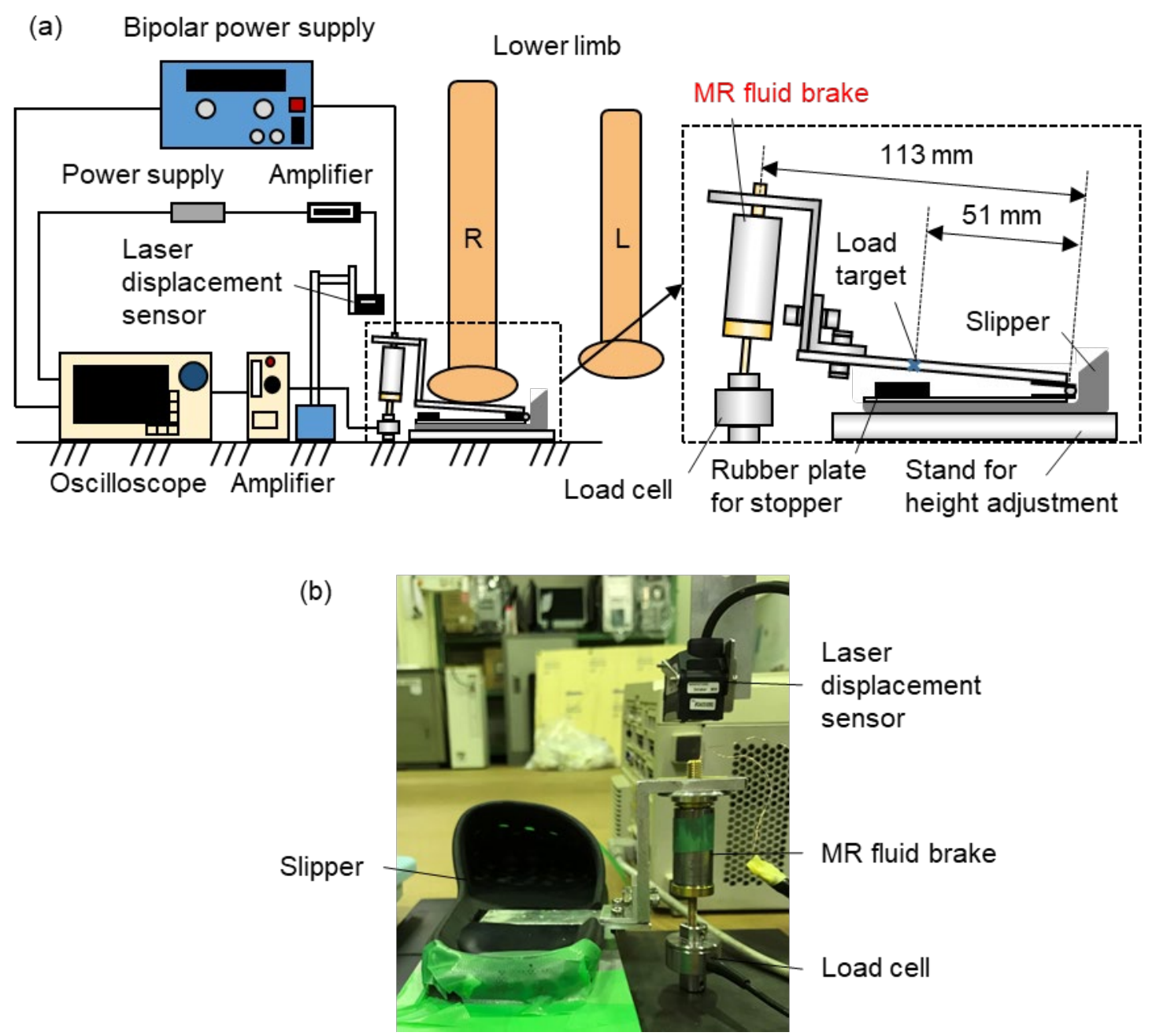
an impact load to the MR fluid brake. (a) Schematic diagram and (b) photograph. The upper part of the slipper was removed on measuring it.

\section{Results and discussion}

The piston moved by approximately $2.5 \mathrm{~mm}$ when a human applied load at $0 \mathrm{~A}$ (Figure 14). In contrast, the piston barely moved, even when a load was applied at 1.5 A (Figure 15). A $2.5 \mathrm{~mm}$ displacement when no electric current was applied was smaller than a $7 \mathrm{~mm}$ stroke. This may be because the load applied to the MR fluid brake was smaller than expected due to the plantar height being given only to a part of the sole. However, although the displacement without electric current was smaller than the stroke, the displacement was kept constant against the impact load when the electric current was applied. The device acted as a brake when the electric current was applied.

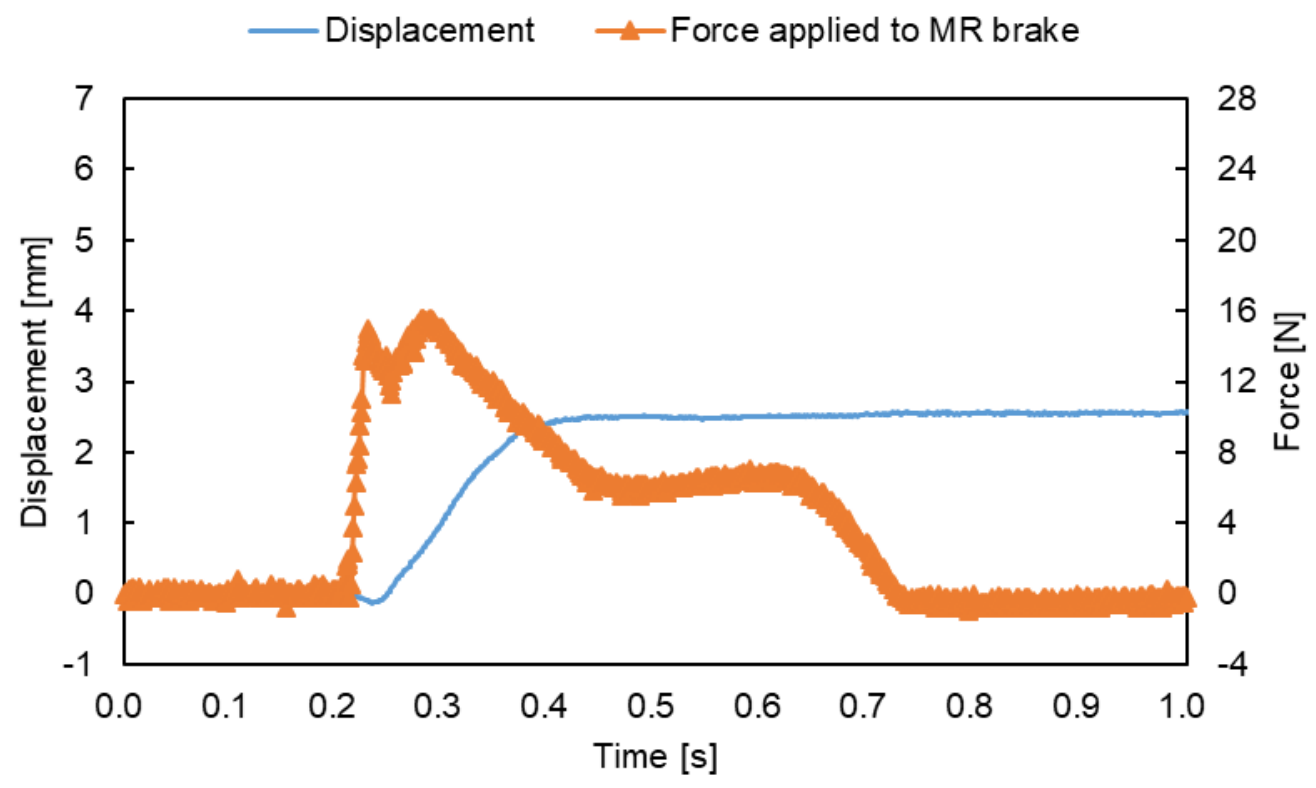

Figure 14 Time series of the piston displacement and force applied to the MR fluid brake (0 A). 


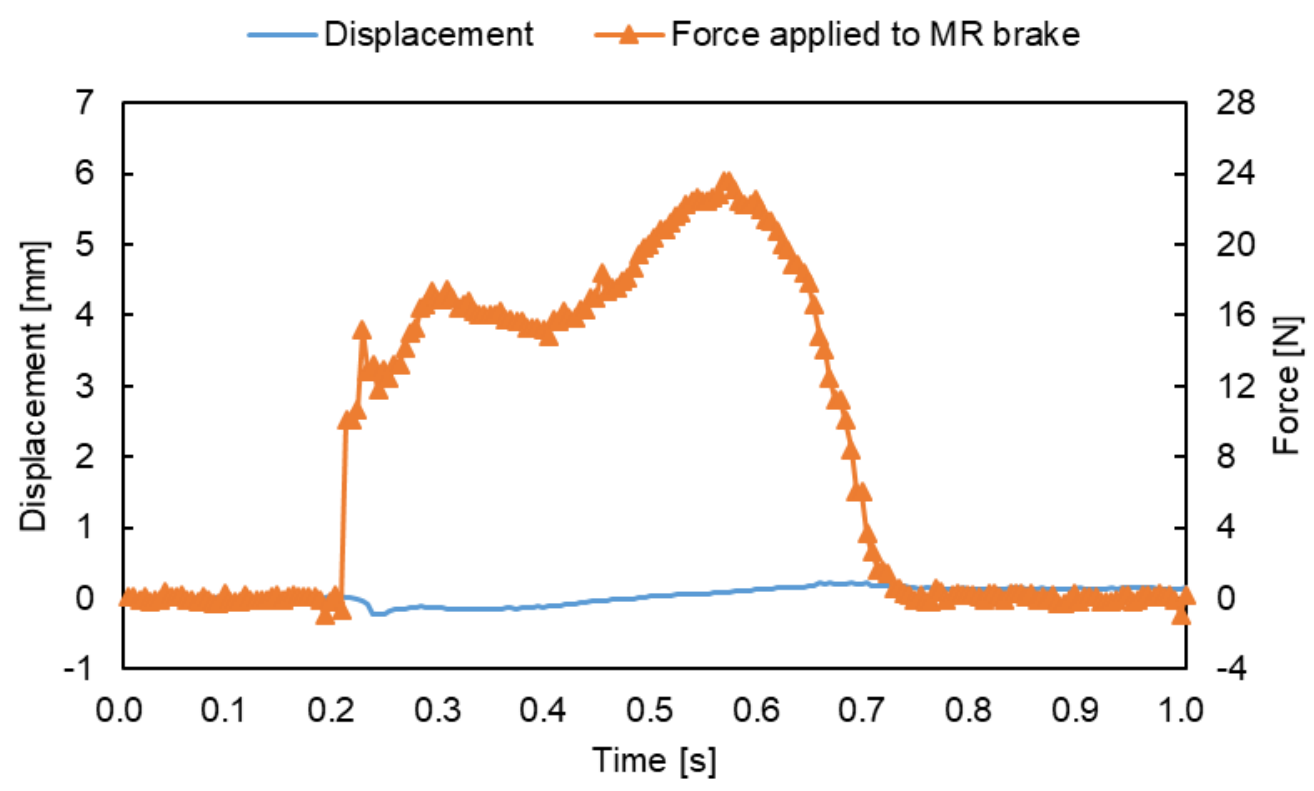

Figure 15 Time series of the piston displacement and force applied to the MR fluid brake (1.5 A).

\section{Evaluation test of the fall prevention effect of the orthosis}

The COG-COP inclination angle on the frontal plane was measured when walking on a slope. The orthosis effect on preventing falls was evaluated from the minimum $\mathrm{COG}-\mathrm{COP}$ inclination angle during the right-leg stance phase with the attached MR fluid brake.

\subsection{Methods}

Four slopes with a low right side, whose angle was $10^{\circ}$, were prepared. Three of the four slopes were placed above the force plate (Figure 16). Eleven infrared markers were attached to the body, and the position of each marker was measured by a high-precision 3D motion measurement system. The markers were attached to the top of the head, acromion process, buttocks, knee joint, ankle joint, and toes. The COG of each body segment part was determined from the measurement results. The COG position was determined from each body part's mass ratio and center of gravity (Nakamura, Nagasaki, et al., 2005). The COP position on the slope of the first step of the right foot of the slope was determined from the measurement results of the force plate (Figure 17(a)). Finally, the COG-COP inclination angle was calculated from the COG and the COP on the slope (Figure 17(b)). The sampling frequency was $100 \mathrm{~Hz}$ for the 3D motion measurement system and $1000 \mathrm{~Hz}$ for the force plate.

The three conditions are as follows: 1) wearing a fall prevention device without the MR fluid brake, 2) with the MR fluid brake attached and current OFF; and 3) current ON. The condition of the current ON corresponded to the control of giving the plantar height when a risk of falling occurred. 
(a)

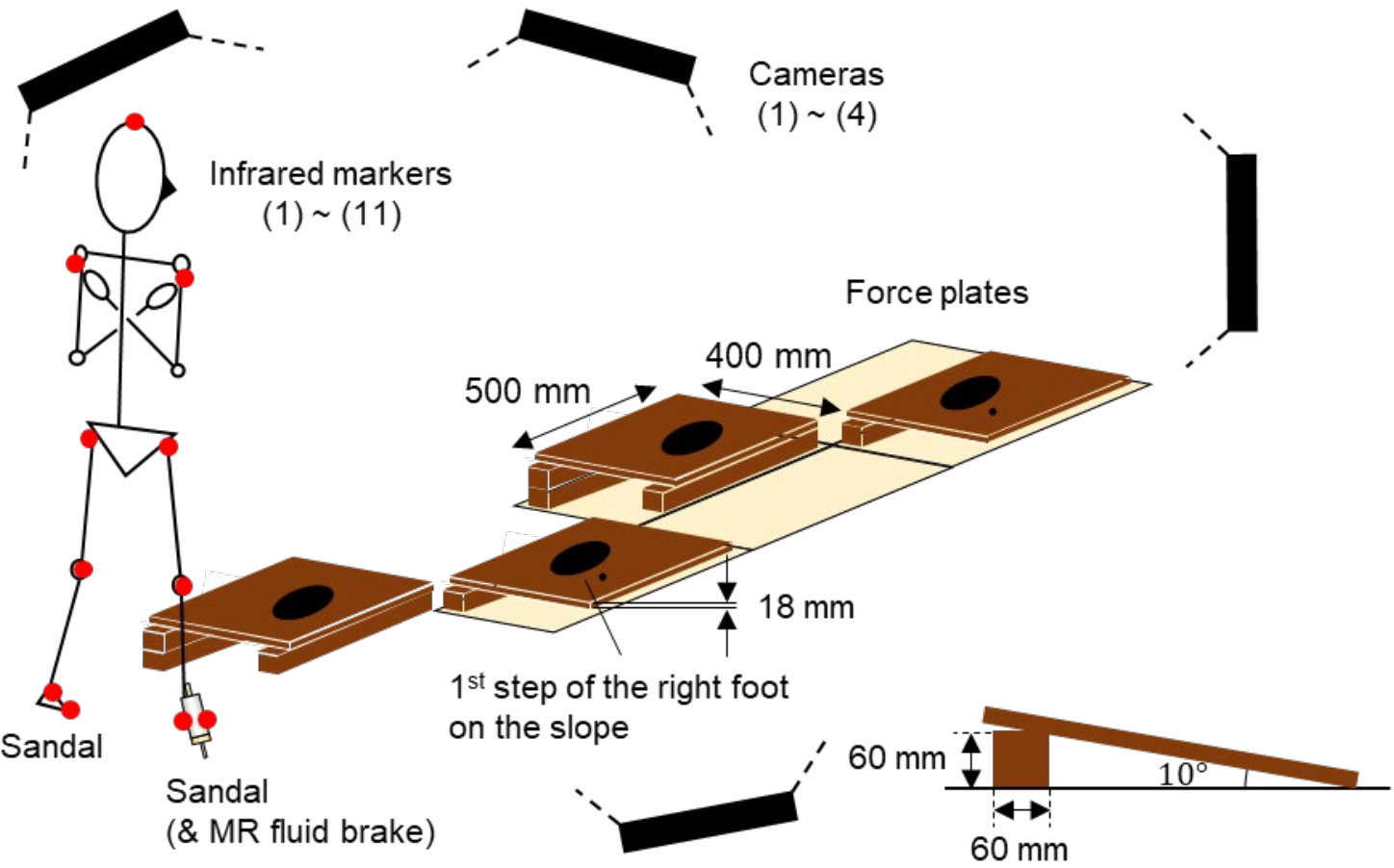

(b)
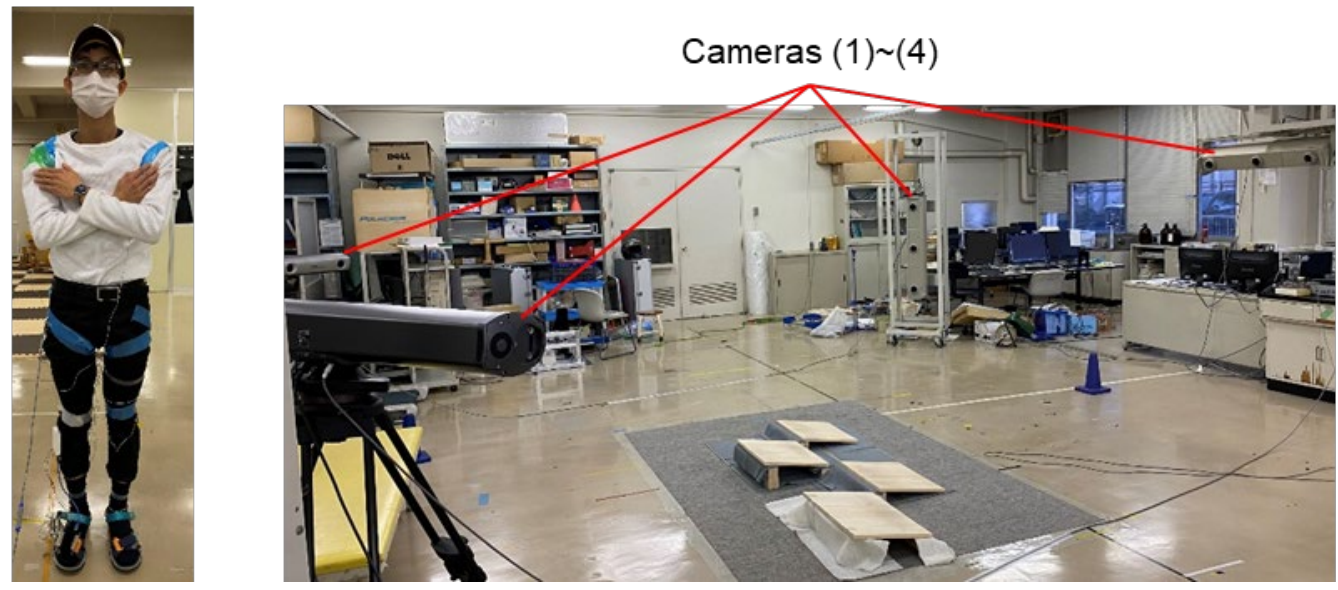

Figure 16

The experimental setup for evaluation test of the fall prevention effect of the orthosis. (a) Schematic and (b) photograph. Lateral slopes are placed on the force plates. Eleven infrared markers are attached to each position on the body. The position of each marker is measured by four cameras. 


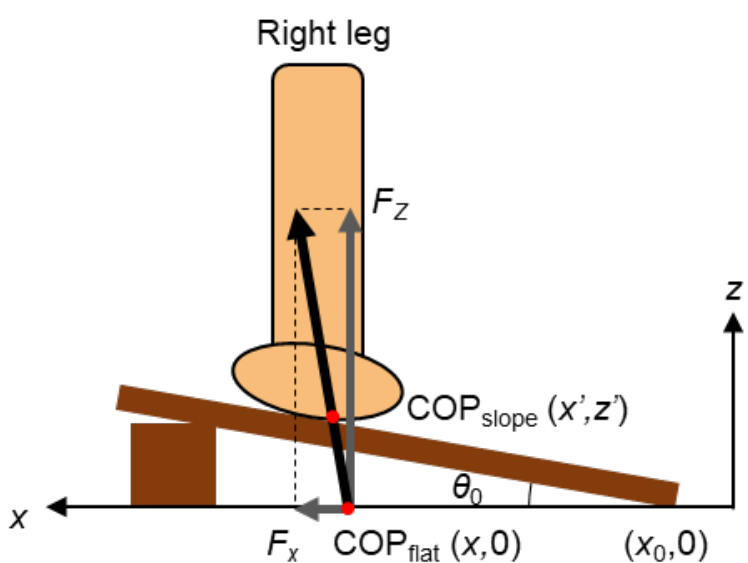

$\mathrm{COP}_{\text {slope }}\left(x^{\prime}, z^{\prime}\right)$

$=\left(\frac{x_{0} \tan \theta_{0}-x \frac{F_{Z}}{F_{x}}}{\tan \theta_{0}-\frac{F_{z}}{F_{x}}}, \frac{\left(x_{0}-x\right) \tan \theta_{0}}{\tan \theta_{0}-\frac{F_{z}}{F_{x}}} \cdot \frac{F_{z}}{F_{x}}\right)$

(a) The COP position on the slope is calculated from the measurement results of the force plate.

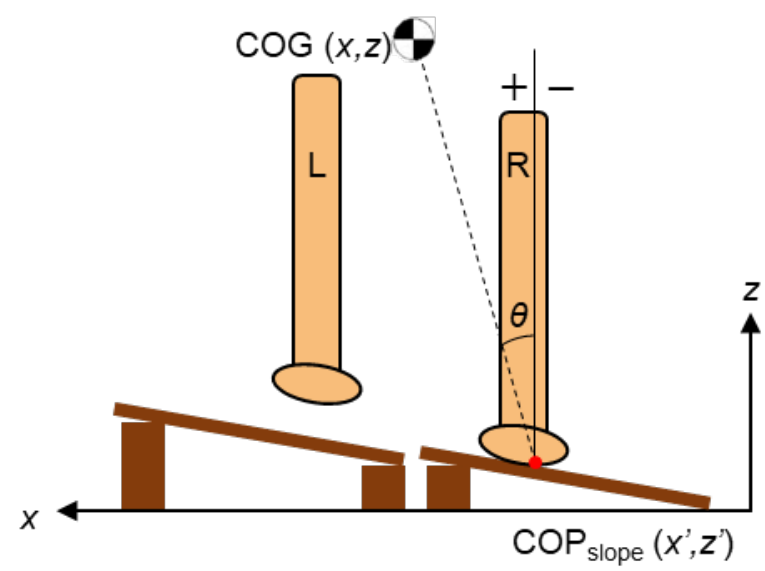

$$
\theta=\tan ^{-1} \frac{x-x^{\prime}}{z-z^{\prime}}
$$

(b) The COG-COP inclination angle is calculated from the position of the COG and the COP on the slope.

Figure 17 Analysis methods for the acquired data.

\subsection{Results and discussion}

The COG-COP inclination angle in the condition without the MR fluid brake, current OFF, and current ON was $4.0^{\circ}, 4.2^{\circ}$, and $4.4^{\circ}$ on average, respectively and they had some variation. There was a significant difference in the angle between current ON and the condition without the MR fluid brake (Figure 18). In a study that examined the effect of the plantar height on fall prevention using a theoretical model that combined a self-righting doll and an inverted pendulum, the COG-COP inclination angle with the MR fluid brake was larger than without it (Morimura, Yamamoto, et al., 2020), which was consistent with the experiment results. In one of trials in the condition without the MR fluid brake, the COG-COP inclination angle was $3.3^{\circ}$ which was very close to the angle in the BOS limit of this subject estimated as $3.2^{\circ}$ from the body measurement and the moment of force to prevent fall little acted on the subject to be almost unstable as shown in Figure 1. On the other hand, the minimum value of trials in current ON was $4.1^{\circ}$ far from the BOS limit angle of $3.2^{\circ}$ and the moment of force to prevent fall acted on the subject to be stable. The results indicate that the orthosis using a compact, light, and power-saving MR fluid brake is effective in preventing lateral falls by increasing the COG-COP inclination angle by giving the plantar height when walking on a slope simulating the falling risk. 


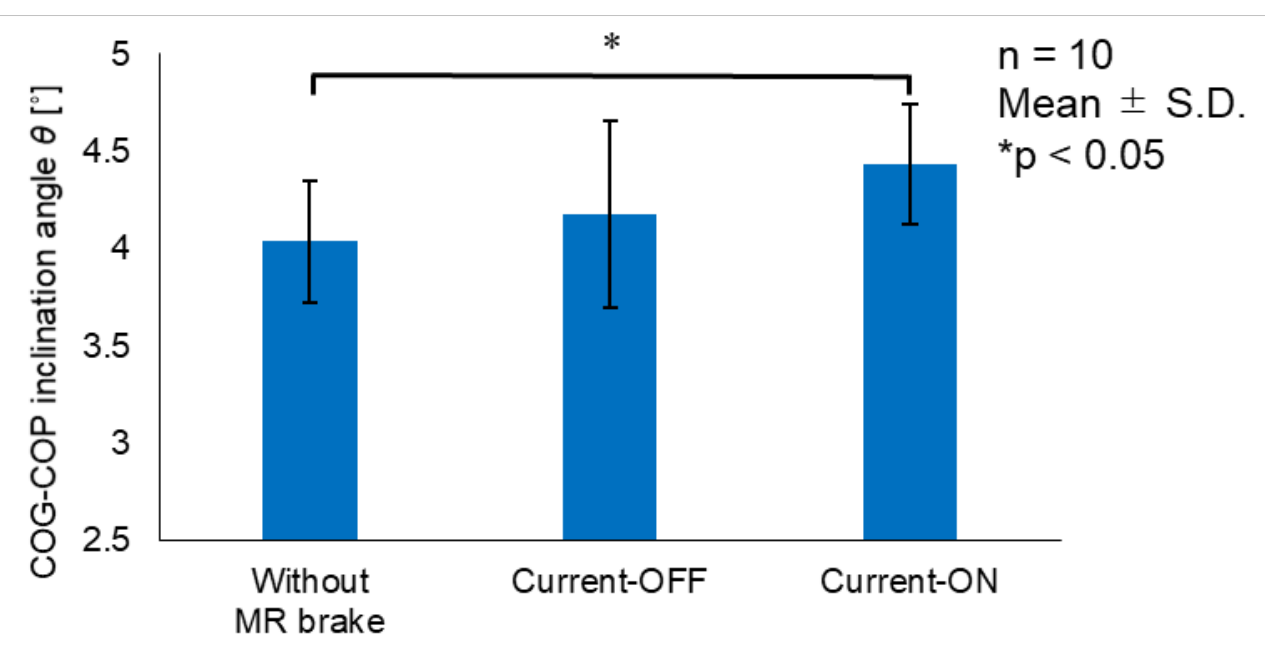

Figure 18 Minimum value of the COG-COP inclination angle during contact of the first step of the right foot on the slope. The condition of the current $\mathrm{ON}$ corresponds to the control of giving the plantar height at the risk of falling.

\section{Conclusion}

This study described the design and fabrication of a compact MR fluid brake capable of maintaining plantar height for use in a lateral fall prevention orthosis, the development of an orthosis incorporating the MR fluid brake, the performance evaluation of the MR fluid brake, and the evaluation of the fall prevention effect of the orthosis. The MR fluid brake was compact, light, and power-saving, generates enough force to support the body weight, and is responsive enough to prevent falls. The MR fluid brake is $88.4 \mathrm{~mm}$ long, has a cylinder outer diameter of $20 \mathrm{~mm}$ and a mass of $128 \mathrm{~g}$, and operates on two AA batteries. The time constant was $179 \mathrm{~ms}$, which is sufficient to prevent falls. When the current was $1.5 \mathrm{~A}$, the dominant generated force was the frictional force amounting to approximately 125 $\mathrm{N}$, which is suitable for maintaining the plantar height. When the current was not applied, the force was approximately $17 \mathrm{~N}$, and the MR fluid brake did not assist walking to improve the residual function during normal walking. In addition, when the MR fluid brake was applied with an electric current, the piston position was maintained at a constant level against the impact load applied by a human walking, indicating that the MR fluid brake is applicable as an actuator for the fall prevention device. When walking on a slope simulating the risk of falling while wearing the developed orthosis, the $\mathrm{COG}-\mathrm{COP}$ inclination angle of a subject in the condition without the MR fluid brake and with the MR fluid brake in the ON state of current was $4.0^{\circ}$ and $4.4^{\circ}$, respectively, and the moment of force acting on the subject to prevent fall was larger and the subject was more stable by the ON-state MR fluid brake.

\section{Acknowledgments}

The authors would like to thank Professor Haruhiko Sato and Mr. Hiroki Tanabe for technical assistance with the experiments. 


\section{Funding}

Part of this work was supported by "YNU Diversity Research Grant" under MEXT Funds for the Development of Human Resources in Science and Technology, Japan, "Initiative for Realizing Diversity in the Research Environment (Collaboration Type)."

Part of this work was supported by the Joint Research Promotion Program of the Research Institute of Environment and Information Sciences, Yokohama National University, Japan, Joint Research Project "Type C".

\section{References}

Andrew, P. D., Arima, K. and Hidaka, M. (2018). Kinesiology of the Muscukoskeletal System, Ishiyaku Publisher, Inc.

Barak, Y., Wagenaar, R. C. and Holt, K. G. (2006). Gait Characteristics of Elderly People with a History of Falls: a Dynamic Approach. Physical Therapy, 86(11), pp. 1501-1510.

Bavdek, R., Zdolšek, A., Strojnik, V. and Dolenec, A. (2018). Peroneal Muscle Activity during Different Types of Walking. Journal of Foot and Ankle Research, 11(50), 50.

Berger, W., Trippel, M., Discher, M. and Dietz, V. (1992). Influence of Subjects Height on the Stabilization of Posture. Acta Oto-Laryngologica, 112(1), pp. 22-30.

Cummings, S. R. and Nevitt, M. C. (1989). A Hypothesis: the Causes of Hip Fractures. Journal of Gerontology, 44(4), pp. M107-M111.

Iritani, M. (2017). Iritani siki sokuteiban-Kisohen- Publisher of motion and medical.

Ishiguro, T., Tsuboshima, S. and Miyagawa, S. (1978). Kouchokumagunetto no sekkei to ouyou, Ohmsha.

Kaya, B. K., Krebs, D. E. and Riley, P. O. (1998). Dynamic Stability in Elders: Momentum Control in Locomotor ADL. Journals of Gerontology. Series A, Biological Sciences and Medical Sciences, 53(2), pp. M126-M134.

Kobayashi, M. and Shimizu, Y. (2014). Effect of a Foot Insole on Standing Balance -Biomechanical study of the Plantar Arch Function-. Society of Physical Therapy Science, 29(4), pp. 605-607.

Krebs, D. E., Gill-Body, K. M., Riley, P. O. and Parker, S. W. (1993). Double-blind, Placebo-controlled Trial of Rehabilitation for Bilateral Vestibular Hypofunction: Preliminary Report. Otolaryngology - Head and Neck Surgery, 109(4), pp. 735-741.

Krebs, D. E., McGibbon, C. A. and Goldvasser, D. (2001). Analysis of Postural Perturbation Responses. IEEE Transactions on Neural Systems and Rehabilitation Engineering, 9(1), pp. 76-80.

Lee, H. J. and Chou, L. S. (2006). Detection of Gait Instability using the Center of Mass and Center of Pressure Inclination Angles. Archives of Physical Medicine and Rehabilitation, 87(4), pp. 569-575.

MacKinnon, C. D. and Winter, D. A. (1993). Control of Whole Body Balance in the Frontal Plane during Human Walking. Journal of Biomechanics, 26(6), pp. 633-644.

Menz, H. B., Lord, S. R. and Fitzpatrick, R. C. (2003). Age-related Differences in Walking Stability. Age and Ageing, 32(2), pp. 137-142.

MLIT (2003). Structures, construction, Ministry of Land, Infrastructure, Transport and Tourism.

Morimura, K., Yamamoto, R. and Shiraishi, T. (2019). A Study of an Orthosis with Controllable Plantar Height by MR Fluid Brake for Fall Prevention of Patients with Walking Disability. In, Proceedings of the Dynamics and Design Conference, 2019.

Murray, M. P., Kory, B. H. and Clarkson, B, H. (1969). Walking Patterns in Healthy Old Men. Journal of Gerontology, 24(2), pp. 169-78.

Naikwadi, A. (2017). Design Optimization of Disk Brake Rotor. International Research Journal of Engineering and Technology, 4(9), pp. 408-413. Available at: https://irjet.net/archives/V4/i9/IRJETV4I970.pdf.

Nakamura, R., Nagasaki, H. and Saitou, H. (2005). Fundamental kinesiology, Ishiyaku Publisher, Inc.

Nanthakumar, A. J. D. and Jancirani, J. (2019). Design Optimization of Magnetorheological Damper Geometry using Response Surface Method for Achieving Maximum Yield Stress. Journal of Mechanical Science and Technology, 33(9), pp. 4319-4329. 
Omuro, K., Shiba, Y., Obuchi, S. and Takahira, N. (2011). Effect of Ankle Weights on EMG Activity of the Semitendinosus and Knee Stability during Walking by Community-dwelling Elderly. Journal of Physical Therapy Science, 26(1), pp. 55-59.

Phu, D. X., Choi, S. B. (2019). Magnetorheological Fluid Based Devices Reported in 2013-2018: MiniReview and Comment on Structural Configurations. Frontiers in Materials, 6(19).

Phu, D. X., Choi, S. B., Lee, Y. S. and Han, M. S. (2014). Design of a New Engine Mount for Vertical and Horizontal Vibration Control Using Magnetorheological Fluid. Smart Materials and Structures, 23(11).

Phu, D. X., Shah, K. and Choi, S. B. (2014). A New Magnetorheological Mount Featured by Changeable Damping Gaps Using a Moved-plate Valve Structure. Smart Materials and Structures, 23(12).

Rabinow, J. (1948). The Magnetic Fluid Clutch. Transactions of the American Institute of Electrical Engineers, 67(2), pp. 1308-1315.

Shiraishi, T. and Morishita, S. (2004). Measurements of Typical Characteristics of MR Fluids and their Application to Design of MR Devices considering Working Modes(696). Japan Society of Mechanical Engineers, 70, pp. 128-134.

Statistic Bureau of Japan (2016). Statistical handbook of Japan 2016. Available at: https://www.stat.go.jp/data/nihon/pdf/16nihon.pdf.

Takai, I., Miyano, M., Nakai, N., Yamaguchi, T., Yoshimura, T., Shirahama, H., Murakami, M., Inoue, K., Tsukazaki, R., et al. (2001). Postural change and Posture Control with Aging. Japan Society of Physiological Anthropology, 6(2), pp. 11-16.

Takata, Y., Takeda, A., Takahashi, H., Iizima, M., Iwamoto, K. and Uchiyama, E. (2016). The Effect of BMZ Insole to Support Cuboid bone on Dynamic Balance Ability in Healthy Adults. Journal of Allied Health Sciences, 7(1), pp. 7-10.

WHO scientific group (2003). Prevention and Management of Osteoporosis, World Health Organization. 